\begin{tabular}{|c|c|c|c|}
\hline Eiszeitalter u. Gegenwart & $\mathbf{3 1}$ & $\begin{array}{c}1-15 \\
16 \mathrm{Abb} .\end{array}$ & Hannover 1981 \\
\hline
\end{tabular}

\title{
Denkmäler und Gedenktafeln von Eiszeitforschern in Mittel-Europa*
}

\author{
MARTIN SCHWARZBACH **) \\ Inventory, monument, memorial tablet, Ice-Age-Researcher, index map. \\ Germany, Switzerland
}

Kurzfassung: Die Geschichte der Eiszeitforschung wird an Hand von Denkmälern und Gedenktafeln in Mittel-Europa (vor allem in der Schweiz) lebendig gemacht, angefangen bei dem Gemsenjäger Perraudin bis hin zu Alfred Wegener und Albrecht Penck.

\section{[Monuments and Memorial Tablets of Ice-Age Researchers in Middle Europe]}

A bstract: The story of ice-age investigation is kept alive by monuments and memorial tablets in Middle Europe (especially in Switzerland). This starts with the chamoishunter PERraudin and ends with Alfred Wegener and Albrecht Penck.

Die Erkenntnis, daß sich das Klima im Laufe der Erdgeschichte geändert habe, geht bis ins 17. Jhdt. zurück, aber die Eiszeitforschung wurde erst um 1800 geboren, in den gletscherreichen Schweizer Alpen. An die frühen Erforscher der Eiszeit erinnern Denkmäler und Gedenktafeln - den meisten heutigen Eiszeitforschern unbekannt. An Hand solcher „Meilensteine der Forschung“ soll im folgenden ein Stück ihrer Geschichte lebendig

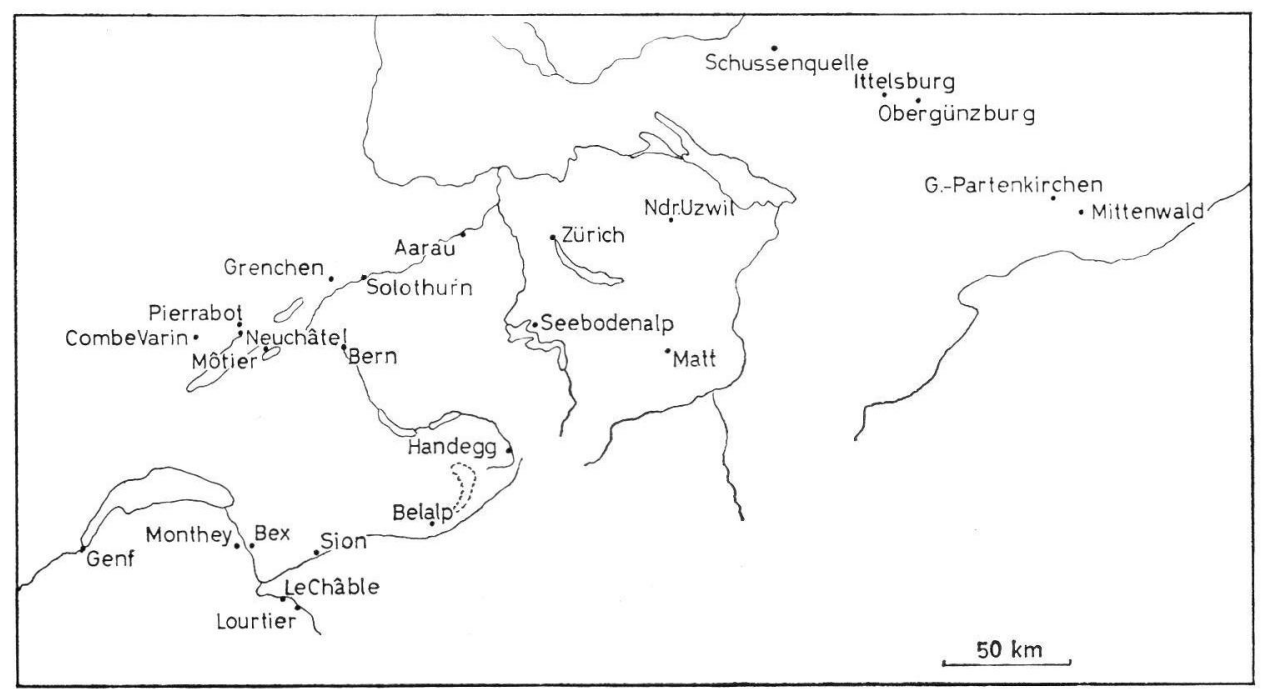

Abb. 1: Úbersichtskarte der Denkmäler und Gedenktafeln im Alpengebiet.

*) Der Aufsatz ist die erweiterte Fassung eines Vortrages auf der Hauptversammlung der Dt. Quartärvereinigung in Aachen, 12.9.1980.

**) Anschrift des Verfassers: Martin $\mathrm{Schw}$ a $\mathrm{rzb}$ a ch, Geologisches Institut der Universität, Zülpicher Str. 49, D-5000 Köln. 
gemacht werden. Die Anregung zu dieser Beschäftigung gaben zwei Aufsätze von HöLDER $(1960,1961)$ über "Geologendenkmäler". Es zeigte sich, daß es davon noch weit mehr gibt, als es zunächst schien. Eine umfangreiche Dokumentation „Auf den Spuren unserer Naturforscher", die nicht nur Geowissenschaftler umfaßt, und detaillierte Angaben umfaßt, ist bei der Wissenschaftlichen Verlagsgesellschaft Stuttgart in Druck.

Die Ausführungen beschränken sich auf Mittel-Europa und im wesentlichen auf das Alpengebiet, vor allem die Schweiz (Abb.1). Die an Gletschern viel ärmeren Ostalpen und auch das nördliche Mittel-Europa haben nur wenig Entsprechendes geliefert.

Ausgangspunkt der Eiszeitforschung waren die „e r r a t is che n Blöcke “, d. h. Felsblöcke, die offensichtlich nicht aus der Gegend stammten, in der sie gefunden wurden. „Nous les désignerons sous le nom de blocs erratiques“, schreibt AleXandre Brongniart 1828 (BöHm v. Böhmersheim 1901: 60, unter Bezugnahme auf Naumann). Aụs dem Futurum „désignerons“ (wir „werden bezeichnen“...) kann man wohl entnehmen, daß BrongNIART einen neuen Begriff in die geologische Nomenklatur einführen wollte. Allerdings wird schon vor ihm von „fremdartigen Geschieben“ und von „Findlingen" gesprochen. Wir werden den „Erratikern“ im folgenden noch mehrmals und aus einem ziemlichen prosaischen Grunde begegnen, denn sie verlockten immer wieder dazu, sie als bequeme und billige Geologen-Denkmäler zu verwenden.

\section{J. P. PER R A U D I N}

Als einer der ersten, der aus den erratischen Blöcken der Alpen auf ehemalige weite Verbreitung der Alpengletscher geschlossen hat, gilt der Walliser Gemsenjäger JEAN Pierre Perraudin (1767-1858) aus dem Bagnes-Tal, einem südlichen Quertal der oberen Rhone. In dem Dorf Le Châble trägt das Gemeindeamt eine Gedenktafel für Perraudin, die ihn voll Stolz, wenn auch etwas übertrieben, als „inventeur de la théorie des glaciers“ bezeichnet. Le Châble war nicht sein Geburts- oder Wohnort; das war vielmehr das wunderschöne Walliser Dörfchen Lourtier, etwas talaufwärts gelegen und zur Gemeinde Le Châble gehörig.
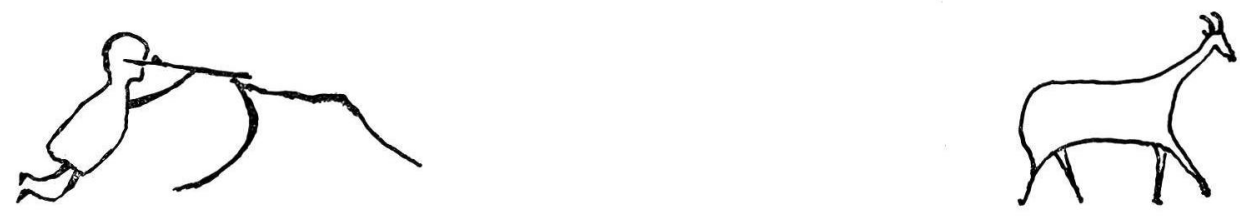

Abb. 2: J. Perraudin als Gemsenjäger - von ihm selbst in einen Holzbalken seines Hauses in Lourtier eingeritzt. Länge ca. $40 \mathrm{~cm}$. Nach einem Foto 1979.

Das Geburtshaus in Lourtier ist erhalten, trägt aber keine Gedenktafel. Wohl aber enthält es neben einigen anderen Erinnerungsstücken ein einmaliges Selbstbildnis, wenn man so sagen darf. Perraudin war nämlich auch ein geschickter Zimmermann gewesen und hatte in einem Deckenbalken seiner Werkstatt eine Zeichnung eingeritzt, die ihn selber auf der Gemsenjagd zeigt, „knieend aufgelegt“, um im Jargon der Schützen zu sprechen. Die Quartärforscher werden unwillkürlich an prähistorische oder altägyptische Zeichnungen denken. Es ist mir nicht bekannt, ob die Zeichnung jemals in der Literatur erwähnt wurde. Eine freundliche Nachfahrin des alten PERRAudin zeigte sie mir 1979 (Abb. 2). 


\section{VENETZ}

Von Perraudin geht, wenn man den Vergleich gebrauchen darf, eine StammbaumLinie zu Ignace Venetz, von diesem zu Jean de Charpentier, und schließlich zu Louis Agassiz. Jeder von diesen hat von seinem Vorgänger die glaziale Deutung der erratischen Blöcke übernommen, sie zuerst nicht geglaubt, jedoch kritisch nachgeprüft und am Ende für richtig befunden und weitergegeben (Abb. 3).

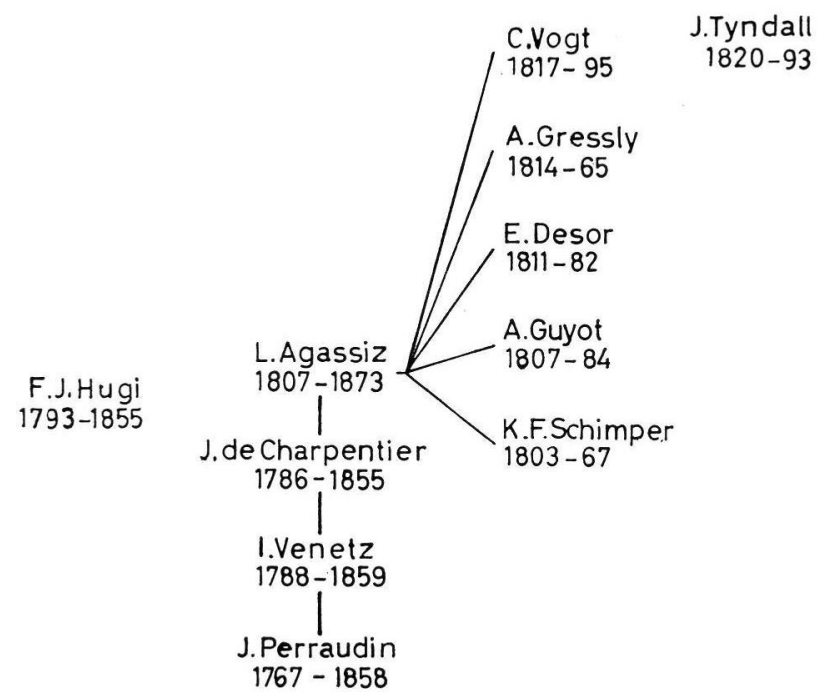

Abb. 3: „Stammbaum“ der frühen Eiszeitforschung in der Schweiz.

Die Beziehungen zwischen Perraudin und Venetz sind allerdings nicht dokumentiert, müssen aber angenommen werden, denn der Walliser Wege- und Brückenbauingenieur VENETZ (1788-1859) hat auch im Bagnes-Tal gewirkt, vor allem 1818, als dort eine Gletschersee-Katastrophe größten Ausmaßes bevorstand. Venetz konnte sie nicht ganz, aber doch weitgehend in engster Zusammenarbeit mit den Talbewohnern verhindern (siehe MariétAn 1959: 45, und Balmer 1970).

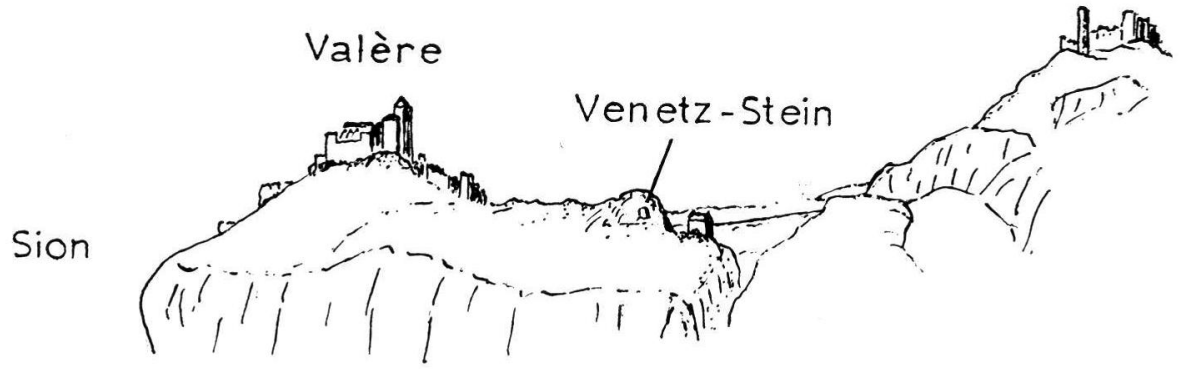

Abb. 4 : Lage des Venetz-Steins in Sion (Sitten). 
VENETZ lebte lange Jahre in Sion (Sitten). 1821 machte er seine glaziologischen Untersuchungen zum ersten Mal bekannt. Ein erratischer Block mit seinem Namen (1868) leider kaum noch leserlich - liegt hoch über Sion, östlich von der Burg Valeria (Abb. 4).

\section{J. De Charpentier}

Sein bedeutender "Schüler“ (ein wenig älter als Venetz) war Jean DE Charpentier (1786-1855), Sohn eines Professors der Freiberger Bergakademie und Student bei ABr. GotTl. Werner, seit 1813 Direktor der Salzbergwerke von Bex an der oberen Rhone; er wohnte im nahen Dörfchen Les Devens.

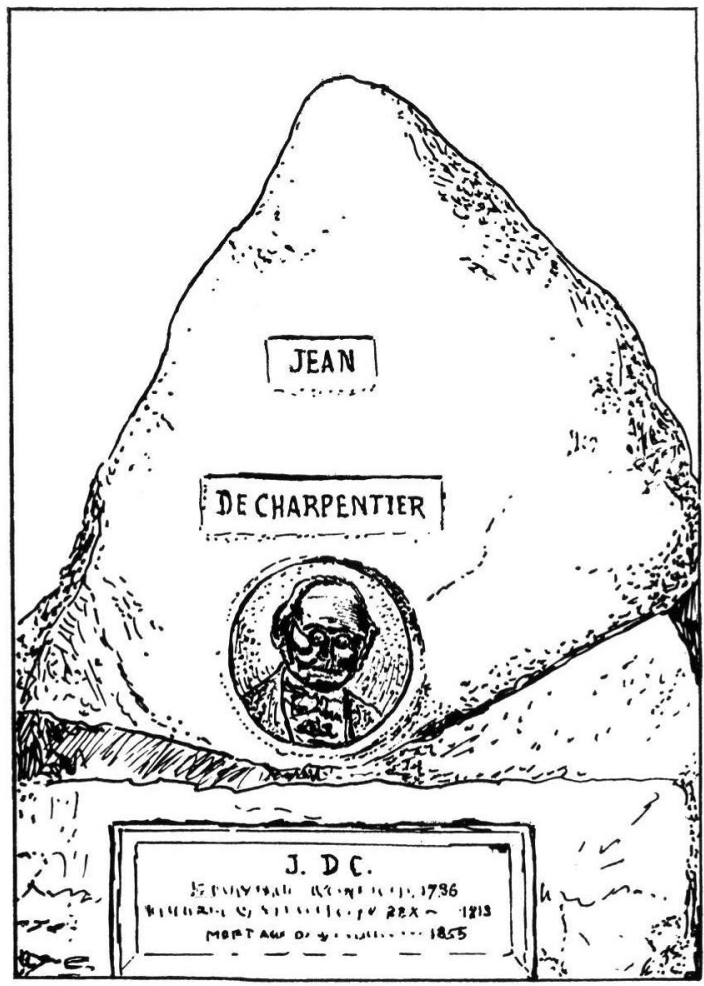

Abb. 5: Denkmal von J. DE Charpentier in Bex. Nach einem Foto gezeichnet.

An der ev. Kirche in Bex errichtete man ihm ein Denkmal (Abb. 5). Es ist ein erratischer Block, an dem man die Inschriftafel seines Grabes befestigte und durch ein Relief seines Kopfes ergänzte, das RaphaEL Lugeon geschaffen hat, ein Bruder des berühmten Alpentektonikers Maurice Lugeon. Auch ein anderer, viel größerer erratischer Block, die Pierre à Dzo im nahen Monthey, einst vom Kanton Wallis Charpentier geschenkt, trägt seinen Namen und den von Perraudin (Renevier 1877; Schwarzbach 1976a).

\section{A g A S I Z}

Als Gast von Charpentier lebte monatelang bei Bex der junge Louis Agassiz (180773), der sich hauptsächlich mit rezenten und fossilen Fischen beschäftigte, aber von der damals aufkommenden Eiszeitlehre begeistert war. Er arbeitete sich mit eigenen Ideen 
in diese neue Richtung ein, verstand es vor allem auch, andere dafür zu gewinnen, wurde ein Führer der damaligen Eiszeitforschung und Neuchâtel durch ihn deren eigentlicher Ausgangspunkt. In der alten Universität steht seine Büste (Abb.6) und nicht weit von der

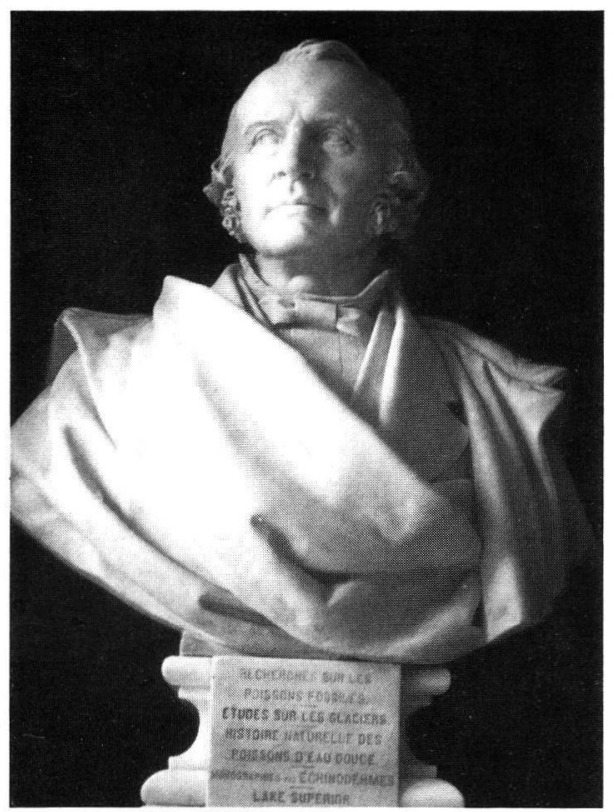

Abb. 6: L. Agassiz. Büste in der Universität Neuchâtel. fot. 1980.

Universität das große Museumsgebäude, in welchem der erst Dreißigjährige am 25. 7. 1837 in seiner Eröffnungsansprache als Präsident der Schweizerischen Naturforsch. Gesellschaft die „Eiszeit begründete“, wie mir EUGEN WEGMANN einmal scherzhaft schrieb (Abb. 7).

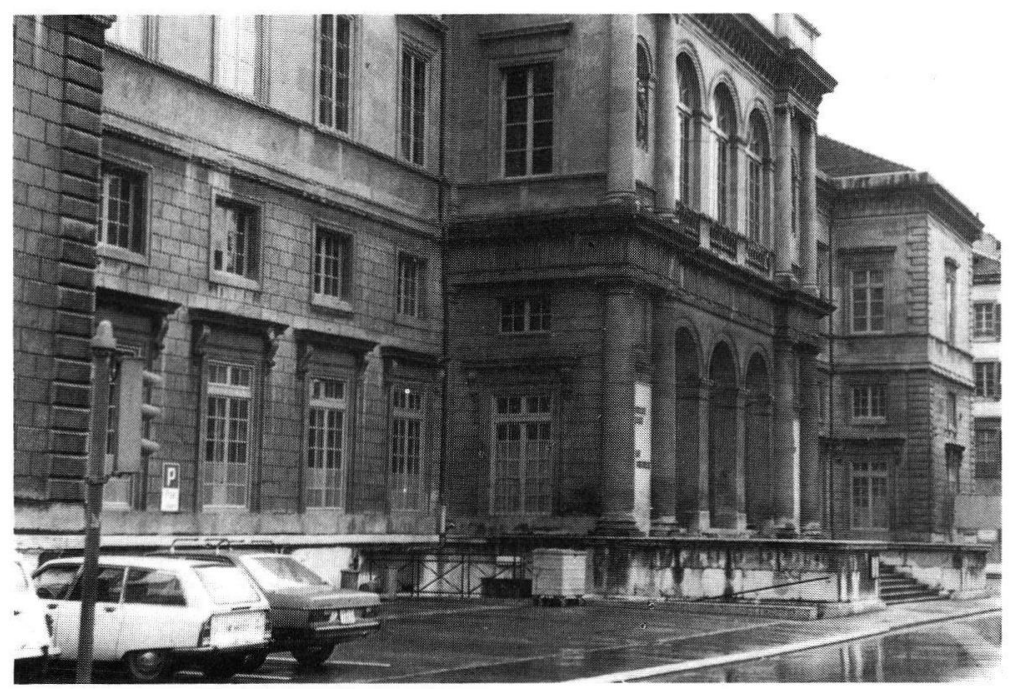

Abb. 7: Das Museumsgebäude in Neuchâtel, in dem L. Agassız 1837 „die Eiszeit begründete“. Foto von E. WEGMANN freundlicherweise übermittelt. 
Agassiz verlas bei dieser Gelegenheit auch eine Abhandlung von Schimper über die Eiszeit; dessen Ode, in der dieser neue Begriff zum ersten Mal literarisch in Erscheinung trat, hatte Schimper bereits am 15. 2. 1837 (seinem und Galilei's Geburtstag) in Neuchâtel verteilt.

Ein berühmter Teilnehmer der Tagung war Leopold v. Buch (1774-1853) - ein erbitterter Gegner der „Eiszeit". Trotzdem sei bei dem Thema „Eiszeitforscher" auf das eindrucksvolle Denkmal hingewiesen, das dem sonst so verdienten Geologen in den Ostalpen (bei Gr. Raming) errichtet wurde: ein gewaltiger „erratischer“ Block - aber ein nicht glazial, sondern tektonisch verfrachteter.

AGAssiz hat eine zusammenfassende Darstellung der Gletscher-Untersuchungen (1841) mit „Gefühlen der Achtung und Freundschaft“ Venetz und Charpentier gewidmet. Eine unmittelbare Erinnerung an seine Beobachtungen stellt die Inschrift „1838 L. Agassiz Eisschliff“ dar, die damals in eisgeschliffenen Granit der „Helle Platten“ zwischen Handeck und Grimsel-Hospiz eingehauen wurde (Abb. in BALMER 1975: 16). Heute würde man das allerdings wohl als Umweltverschandelung bezeichnen!

Von Agassiz gibt es noch mehr steinerne Spuren, so eine Gedenktafel an seinem Geburtshaus, dem Pfarrhaus von Môtier am Murtensee. Ein großer Findling bei Môtier und vor allem ein gewaltiger Block von Montblanc-Granit bei Neuchâtel tragen seinen Namen. Der letztere Block heißt Pi er rabot (eigentlich Pierre à Crapaud = Krötenstein) (Abb. 8). Er ist einer der berühmtesten erratischen Blöcke. Schon der schottische Eiszeit-

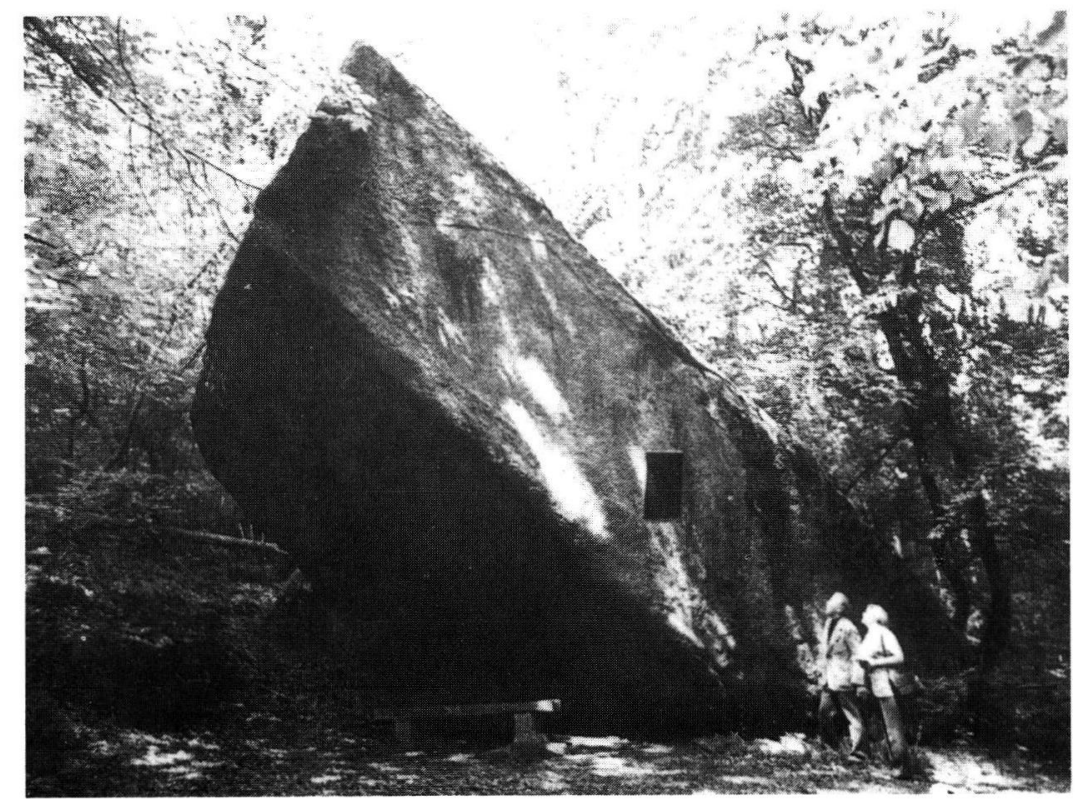

Abb. 8: Pierrabot bei Neuchâtel. Die Gedenktafel an dem erratischen Block von Montblanc-Granit enthält die Namen von Agassiz, Guyot, Desor, Dupasqier. fot. 1980.

pionier John Playfair hat ihn erwähnt, und noch bemerkenswerter ist dieser Felsblock deswegen, weil er bereits 1838 auf Vorschlag von AgAssiz unter Naturschutz gestellt und durch einen Waldpfad zugänglich gemacht wurde. AgAssiz ging 1846 nach Nordamerika und wurde dort hochangesehener Professor an der Harvard-Universität; er ruht nun unter 
einem Findling vom Unteraar-Gletscher auf dem Mt. Auburn-Friedhof von Cambridge, Mass. Ein sonderbares Schicksal hatte eine Agassiz-Statue in der kalifornischen StanfordUniversität: beim Erdbeben von San Francisco 1906 stürzte sie vom Sockel und verlor ihren Kopf (Abb. in JaCOPI 1969 und SchwarZBACH 1976b).

\section{A. $\mathrm{G} U$ Y O T}

Die Tafel an der Pierrabot - 1966 anstelle der alten Beschriftung auf Anregung von J.-P. Portmann angebracht - führt außer "AGAssiz" noch drei weitere Namen an, die mit Neuchâtel verbunden waren: Guyot, Desor und Dupasquier. Guyot und Desor gehörten zu den engen Mitarbeitern von AGAssiz, und beide folgten ihm bald in die USA.

Auch Arnold Guyot (1807-84) wurde Professor in Amerika, an der Princeton University. Durch einen bedeutenden Nachfolger von ihm in Princeton, Harry H. Hess, ist der Name "Guyot" ein Jahrhundert später in die internationale geologisch-geographische Nomenklatur eingegangen; auf Anregung von Hess (Ozeanograph, „Plattentektoniker“, im 2. Weltkrieg U-Boot-Kommandant) werden submarine, oben abgeflachte Vulkanberge,

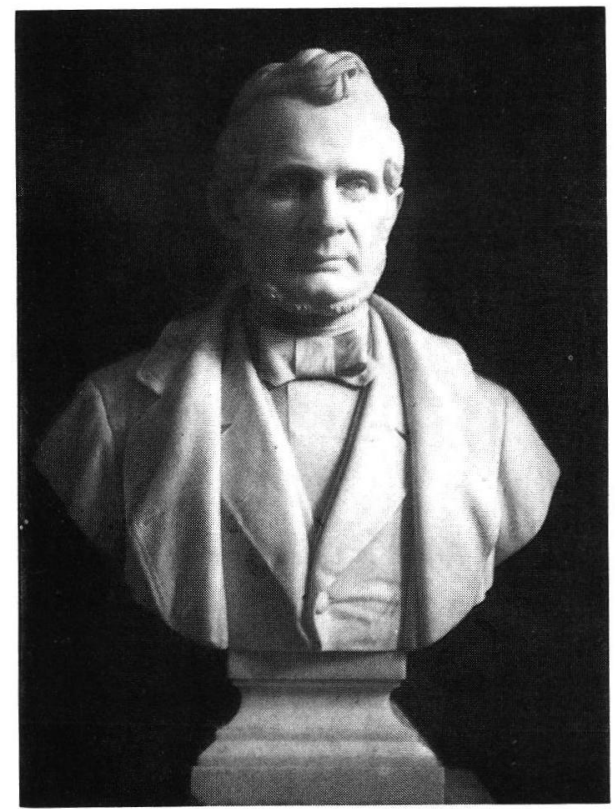

Abb. 9: A. GuYot. Büste in der Univ. Neuchâtel. fot. 1980.

wie sie besonders im Pazifik vorkommen, heute als Guyots bezeichnet. Auch von GuYot steht eine Büste in der alten Universität von Neuchâtel (Abb. 9).

\section{E. DESOR}

Der andere Mitarbeiter von AgAssiz, der mit ihm nach Amerika reiste, war EdouARD Desor (1811-82). Er entstammte (wie J. DE ChARpentier) einer Hugenottenfamilie in Deutschland und wurde in Friedrichsdorf in Hessen geboren. Sein enges Verhältnis zu Agassiz ging später in die Brüche, und so kehrte er 1852 in die Schweiz zurück, wo er bei Neuchâtel einen Landsitz geerbt hatte, Combe Varin (im Jura-Hochtal Les Ponts; Abb. 10). 


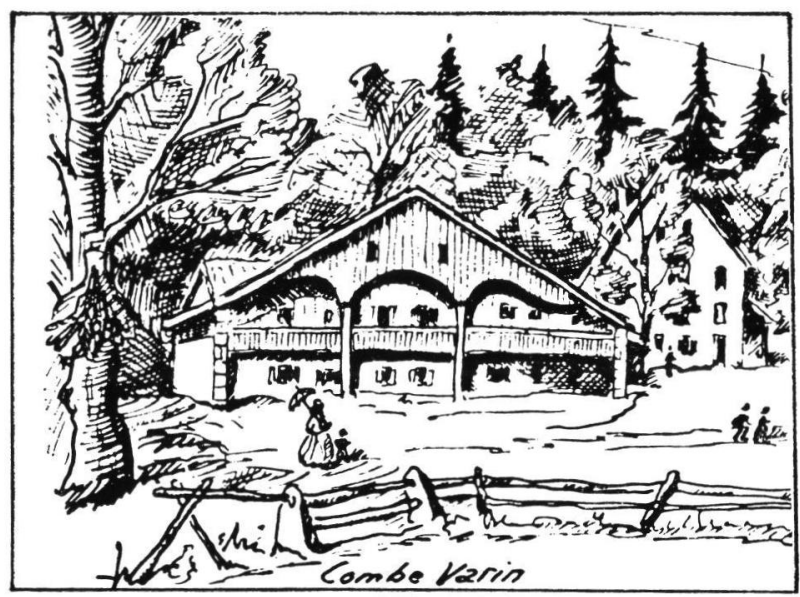

Abb. 10: Combe Varin, der Sommersitz von E. Desor. Titelzeichnung der Zeitschrift Le Petit Rameau de Sapin, Neuchâtel, Mai/Juni 1973.

Dort lebte er im Sommer, und dorthin lud er zahlreiche Gelehrte ein, nicht nur Eiszeitforscher. Er legte sich ein ungewöhnliches „Gästebuch“ an, indem er die Namen illustrer Besucher auf die Rinde von Bäumen einer Allee aufmalte, und da man die weiße Beschriftung bis heute pietätvoll immer wieder erneuerte, existiert diese originelle Allee mit 80 Namen noch immer.

Von Forschern, die sich auch mit der Eiszeit beschäftigten, sind hier u. a. A. Gressly, Guyot, O. Heer, Lyell, F. Roemer, Tyndall, C. Vogt verzeichnet.

Der weitere Kreis um Agassiz

Das ungewöhnliche Ansehen, dessen sich Agassiz erfreute, geht nicht zuletzt daraus hervor, daß man auch einer Reihe seiner Mitarbeiter Denkmäler oder Gedenktafeln widmete. An Guyot und Desor wurde schon erinnert, die Namen von Gressly und C. Vogt tauchten eben in Combe Varin auf. Amanz Gressly (1814-65; Gedenktafeln in Bärschwil, Laufen, bei Solothurn) ist freilich mehr bekannt dadurch, daß er den geologischen Begriff „Fazies“ schuf.

Der Zoologe Carl VOGT (1817-95), in Gießen geboren, fand zweimal in den unruhigen Jahrzehnten des vorigen Jahrhunderts Zuflucht in der Schweiz, wo er schließlich Professor in Genf wurde. Vor der dortigen Universität steht seine Büste. Der streitbare VOGT hat das Mißfallen von KarL MARx erregt; in einem dicken Buch (1860) hat dieser den „kugelrunden“, „hündisch-infamen Verleumder" VOGT mit ausgesuchten Beschimpfungen belegt.

Karl Friedrich Schimper (1803-67), der den Begriff „Eiszeit“ in die Literatur einführte, wurde bereits bei AGAssız erwähnt. Damals verfeindeten sich beide nach einem unerfreulichen Prioritätsstreit für immer. Eine Büste Schimper's steht auf seinem Grab in Schwetzingen bei Heidelberg (Abb. 11), eine Gedenktafel wurde an seinem Sterbehaus angebracht. Seine erste Verlobte hat den bei einem Überfall schwer Verletzten dort zuletzt gepflegt. 


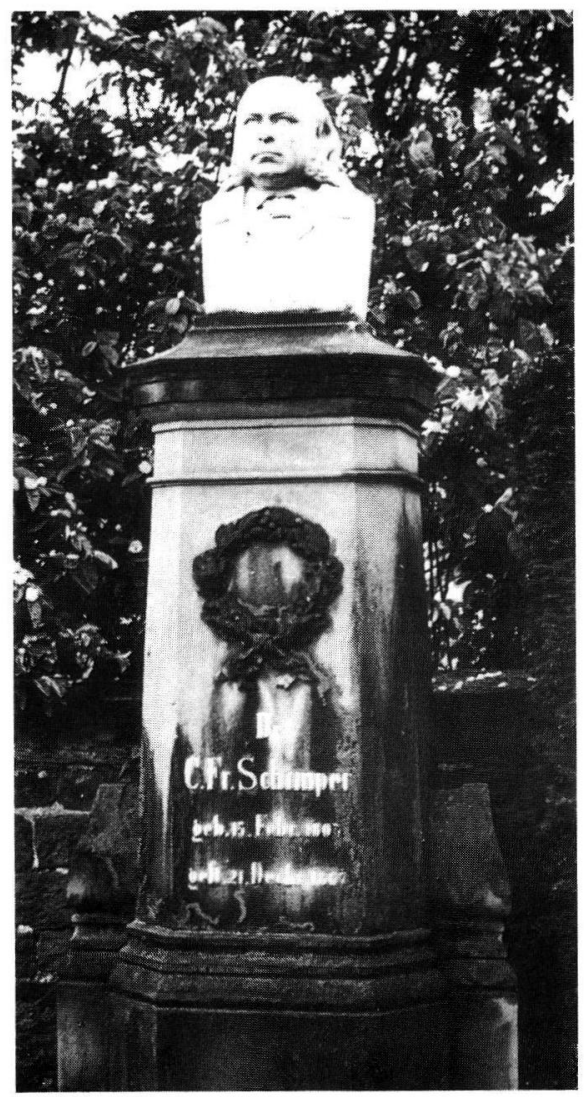

Abb. 11: C. F. Schimper, der 1837 den Begriff „Eiszeit“ in die Literatur einführte. fot. 1974.

Schimper war hauptsächlich Botaniker, Agassiz Zoologe, ebenso Carl Vogt; Charpentier war Bergmann. Es ist aufschlußreich, wie vielseitig der Kreis war, der von der "Eiszeit“ und nicht zuletzt Agassiz's Initiative begeistert — die eigentliche Eiszeitforschung begründete. Ebenso interessant ist es, daß das zwar in der Schweiz vor sich ging, aber nicht wenige Forscher keine gebürtigen Schweizer waren: Charpentier, Schimper, Desor, Vogt. Allerdings sind drei davon in dem Land, das die großzügig aufnahm, dann eingebürgert worden. Andererseits wurden AGAssiz und GuYOT später Amerikaner.

\section{F. J. Hu G I}

Ein Forscher, der wie Agassiz und sogar schon vor ihm mit einer Forschergruppe a u dem Gletscher systematische Beobachtungen anstellte, war Franz Josef Hugi (17931855), geboren in Grenchen, wo ihm die Bürger einen Gedenkstein errichteten (Abb. 12); dann im nahen Solothurn tätig, dessen Museum er gründete. Im Alpinen Museum Bern hängt ein hübsches Bild, das Hugr's Gruppe bei der Arbeit im Gelände zeigt (BUDMiger 1980; ein ähnliches Gemälde: AgAssız mit seinem berühmten „Hôtel des Neuchâtelois“ auf dem Unteraar-Gletscher, besitzt das Geologische Institut Neuchâtel). Hugr arbeitete 


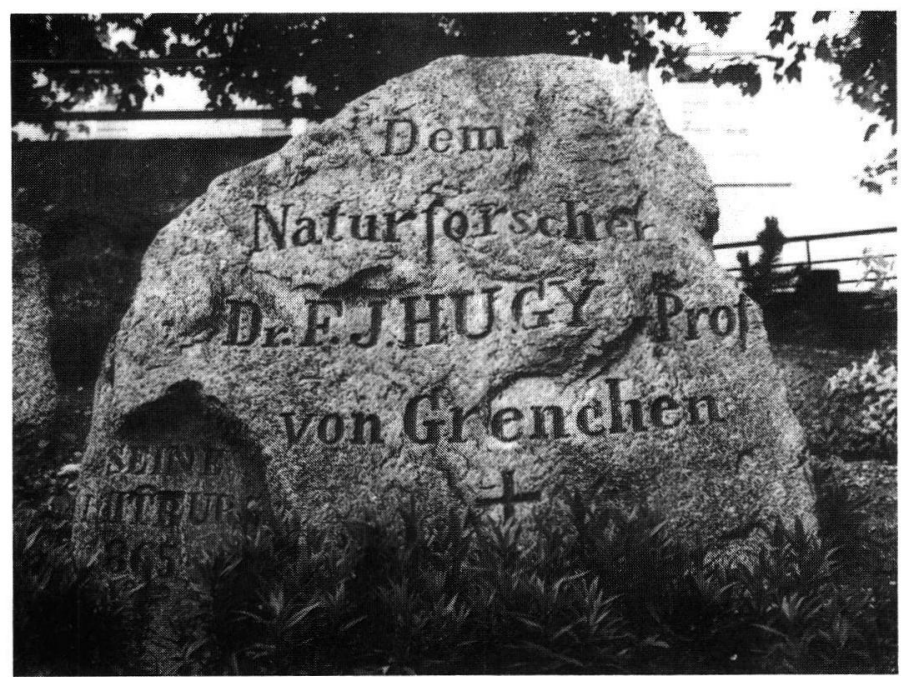

Abb. 12: F. J. Hugr. Gedenkstein in Grenchen (Kt. Solothurn). fot. 1980.

etwas abseits von Agassiz, war auch keineswegs mit allen Ansichten des Neuchâteler Professors einverstanden.

Uberhaupt muß betont werden, daß die Vorstellungen, welche die Eiszeitforscher jener Zeit hatten, zwar revolutionär wirkten und eine neue Epoche paläoklimatologischer Forschung einleiteten, aber in mancher Beziehung längst überholt sind. Das gilt z. B. auch von dem neugeschaffenen Begriff „Eiszeit" selbst, der bei Schimper und Agassiz eine andere Bedeutung hatte als heute bei uns.

\section{Englische Eiszeitforscher in den Alpen}

$\mathrm{Zu}$ den Ausländern, die in jener Zeit an Schweizer Gletschern exakte Untersuchungen durchführten, gehörte (neben dem Schotten J. D. FORBES) der englische Physiker JoHN Tyndall (1820-93), der auch in Deutschland studiert hatte, und in der Schweiz mit Recht durch einen schön gelegenen Gedenkstein über dem Aletsch-Gletscher, oberhalb Belalp, geehrt wurde. Auch das Haus, das er sich bei Belalp bauen ließ, ist erhalten.

Sein Landsmann Charles Lyell (1797-1875), der die Verfrachtung der nordischen Findlinge durch Eisberg-Transporte erklärte, ist in Combe Varin verewigt.

\section{Spätere Eiszeitforscher aus dem Alpengebiet}

Erst in der 2. Hälfte des vorigen Jahrhunderts begann die moderne Eiszeitforschung, die ebenfalls im Alpengebiet durch einige Denkmäler dokumentiert ist.

Detaillierte Moränen-Untersuchungen gehen auf FRIEDRICH MüHLBERG (1840-1915) zurück, Gymnasialprofessor in Aarau westlich Zürich. Seine Büste steht vor der Kantonatsschule, wo er lehrte, und dessen Schüler 1895/96 auch Albert EINSTEIN gewesen ist. Dann ist zu nennen der vielseitige Altmeister der Schweizer Geologen und Verfasser einer "Gletscherkunde“ (1885) Albert Heim (1849-1937) mit einem ihm gewidmeten erratischen Block auf der Seebodenalp über Küßnacht, am Abhang des Rigi, und ein anderer großer Meister, Albrecht Penck (1858-1945), Professor in Wien und Berlin, auf den eine 


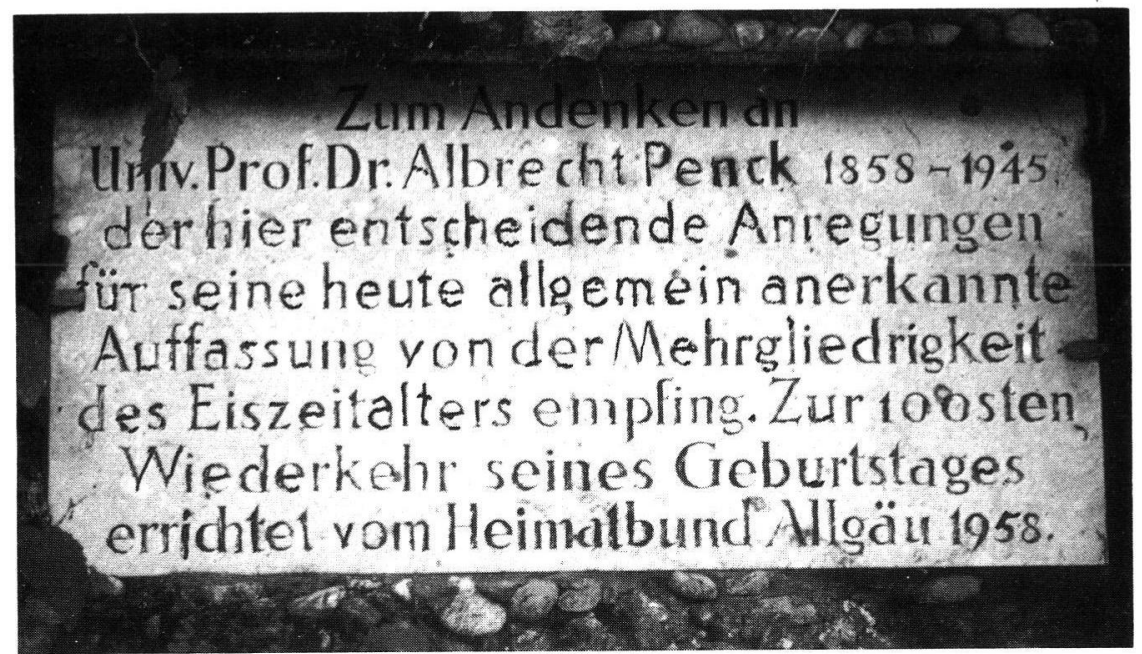

Abb. 13: Gedenktafel für Albr. Penck bei Ittelsburg im bayr. Alpenvorland. fot. 1978.

bescheidene Gedenktafel am Falken bei Ittelsburg (südlich Memmingen) hinweist (Abb. 13), die, soweit ich feststellen konnte, kaum einer der heutigen Quartärforscher kennt (den Hinweis verdanke ich O. Timmermann Köln); auch am Gasthaus „Gletscherschliff“ südlich von Mittenwald ist der Name Penck's auf einer Tafel genannt. Hier sei der Theound Geologe BARTel Eberl (1883-1960) angefügt, der die klassische Terrassengliederung von Penck und Brückner im bayerischen Alpenvorland erweiterte. Eine Tafel (auf die mich L. Scheuenplug aufmerksam machte) an seinem Wohnhaus, dem Benefiziatenhaus in Obergünzburg südöstl. Memmingen, erinnert an ihn.

Eine zweite Gruppe von Eiszeitforschern aus diesem Raum führt der Schweizer Paläobotaniker und Erforscher der fossilen Polarfloren Oswald Heer (1809-83) an. Mit ihm kommen wir von der regionalen Gletscherforschung bereits zu der viel umfassenderen, globalen Paläoklimatologie. Ein Denkmal HeER's steht im alten Botanischen Garten in Zürich, Gedenktafeln gibt es an seinem Geburtshaus in Nieder-Uzwil (Kt. St. Gallen) und in Matt (Kt. Glarus). - In Partenkirchen weist die Windrose auf dem Grabstein von ERICH v. DrYGALSKI (1865-1949) auf seine erfolgreichen deutschen Expeditionen in den Inlandeisgebieten der Arktis und Antarktis hin, und hier können wir Alfred Wegener (1880-1930) und seinen Schwiegervater WLADIMIR KöPPEN (1846-1940) anschließen, die beide zuletzt im alpenländischen Graz wohnten, aber in ihren paläoklimatologischen Forschungen ebenfalls nicht mehr von den Alpengletschern ausgingen, sondern durch die Kontinentalverschiebungs-Hypothese umstürzende Vorstellungen auch über die großen Klimaschwankungen der Erde entwickelten. Sie machten den Eiszeitforschern außerdem die Strahlungskurven von Milankovitch (1879-1958) zugänglich; dessen Geburtshaus (mit Gedenktafel, auf die mich K. Brunnacker hinwies) steht in Dali, Slovenien, und damit bereits in SE-Europa, gehört also, streng genommen, nicht mehr zu unserem auf Mittel-Europa beschränkten Raum. Bei WEGENER sei zunächst nur seine Büste in der Universität Graz und die im September 1980 enthüllte Gedenktafel an seinem Wohnhaus (Wegenergasse 11) genannt, um mit den Gedenk-Spuren im Alpen-Gebiet zu bleiben. Der Name KöPPEN aber lebt, soviel mir bekannt ist, nur in einem Straßennamen in Hamburg-Gr. Borstel weiter. 
Eiszeitforscher im nördlichen Mittel-Europa (Abb. 14)

Damit sind wir im nördlichen Mittel-Europa, das nicht allzuviel für unser Thema geliefert hat. WEGENER ist allerdings erneut anzuführen (mit einer Gedenkstätte in Zechlinerhütte bei Rheinsberg, DDR, und einer Gedenktafel in Ost-Berlin; Abb. in Schwarzвасн 1980a, b). An erster Stelle müssen wir jedoch den Gedenkstein nennen, der nach

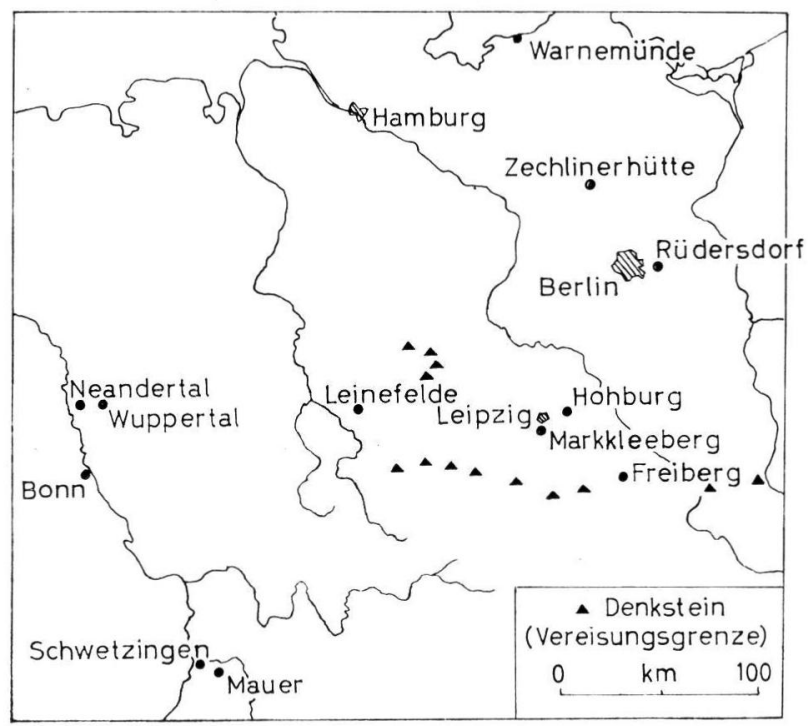

Abb. 14: Übersichtskarte der Denkmäler und Gedenktafeln im außeralpinen Deutschland. Eingetragen sind auch die Denksteine der südl. Vereisungsgrenze in der DDR.

1875 in den Rüdersdorfer Kalkbergen bei Berlin für den Schweden OTto Torell (18281900) errichtet wurde; er kennzeichnet den endgültigen Siegeszug der Gletscherhypothese auch für den norddeutschen Raum.

Von Forschern, die schon früher die Ansicht von Torell in Erwägung zogen, sei Bernh. v. Cotta (1808-79) genannt (Gedenktafel in Freiberg i. Sa.). An den Leipziger Carl Friedr. Naumann $(1797-1873)$ erinnert der "N a u ma n n- He i m-F els s auf dem Kleinen Berg bei Hohburg (nördl. Wurzen, Bez. Leipzig). Seine glattgeschliffenen Flächen wurden zuerst auf Gletscher bezogen, aber sie rühren vom Winde her. (Gletscher-Schliff gibt es aber an benachbarten Stellen auch!). Wichtig war hier vor allem ein Besuch des Schweizers Adolph v. Morlot (1844). L. Eissmann hat das 1974 ausfuhrlich dargelegt. 1870 war auch Albert Heim dort. Der „Naumann-Heim-Fels“ trägt jedoch keine Beschriftung.

Noch mehr würde übrigens A. Bernhardi, Professor an der Forstakademie Dreißigacker bei Meiningen, eine Gedenktafel verdienen; er hat schon 1832 die erratischen Geschiebe Norddeutschlands mit nordischen Gletschern in Beziehung gebracht.

Eine erste gründliche paläontologische Beschreibung nordischer Geschiebe-Fossilien gab Ferdinand Roemer (1818-91), Professor an der Universität Breslau. Ein großer Findling bei Obernigk, nördl. Breslau, trug den Namen „Roemer-Stein“. - Um die quartärgeologische Erforschung Mecklenburgs hat sich EugEN Geinitz (1878-1925) verdient 
gemacht; sein Name wurde auf einem erratischen Block am Steilufer der Ostsee bei Warnemünde (Stoltera) angebracht.

Nicht vergessen seien die Erforscher des e is z e it 1 ichen $\mathrm{M}$ e n s chen. An erster Stelle steht Joh. Carl Fuhlrott (1803/04-77), der Entdecker des ersten Neandertalers, mit einem Gedenkstein in seinem thüringischen Geburtsort Leinefelde (Abb. 15) und Ge-

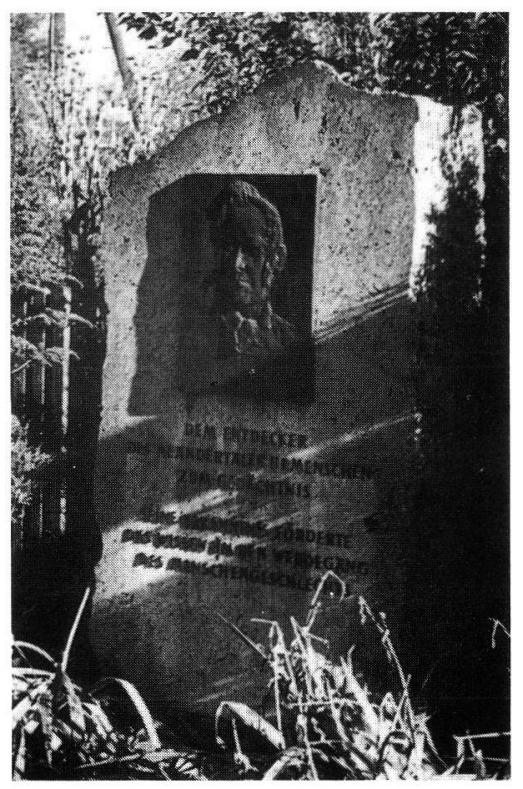

Abb. 15: Denkmal von J. C. FunLrotr, dem Entdecker des Neandertalers 1856; Leinefelde. fot. 1975.

denktafeln an seinem Wohnhaus in Wuppertal-Elberfeld und im Neandertal bei Düsseldorf. Der erste Beschreiber der.Funde war der Bonner Anatom Hermann SchaAfhausen (1816-93): er ruht auf dem erinnerungsreichen alten Friedhof in Bonn. - In seiner Geburtsstadt Regensburg hat der Prähistoriker Hugo Obermaier (1877-1946), dessen Name u. a. mit Altamira verbunden ist, eine Gedenktafel (an der Staatl. Bibliothek).

In Markkleeberg bei Leipzig weist ein Gedenkstein auf die Altsteinzeit-Funde in den Pleiße-Schottern und auf deren Entdecker Franz Etzold (1895) und K. H. Jacob(-FrieSEN) hin (Abb. 16). Gedenksteine an ähnlichen Fundstellen (Mauer b. Heidelberg, Schussenquelle am Federsee u. a.) enthalten keine Hinweise auf die Namen der Erforscher.

Nicht einzelnen Eiszeitforschern, wohl aber der Eiszeitforschung allgemein gelten auch, um das als letztes zu erwähnen, die Denksteine, die in jüngster Zeit besonders auf Anregung von O. Wagenbreth (1978) in der DDR an der S ü dg renze de r ska nd i$\mathrm{n}$ a vis chen Vereis ung errichtet wurden, vom Elbsandsteingebirge über Sachsen und Thüringen bis hin zum Harz (Abb. 14). Das ist ein nachahmenswertes Beispiel für die Bemühungen, wissenschaftliche Erkenntnisse weiteren Kreisen näherzubringen, und es wäre erfreulich, wenn man dies e Grenzmarkierung weiter nach Westen hin fortführen würde - eine Anregung, die bereits 1979 vom Verf. gegeben und auf der Tagung der Deutschen Quartärvereinigung im September 1980 in Aachen erneut ausgesprochen wurde. 


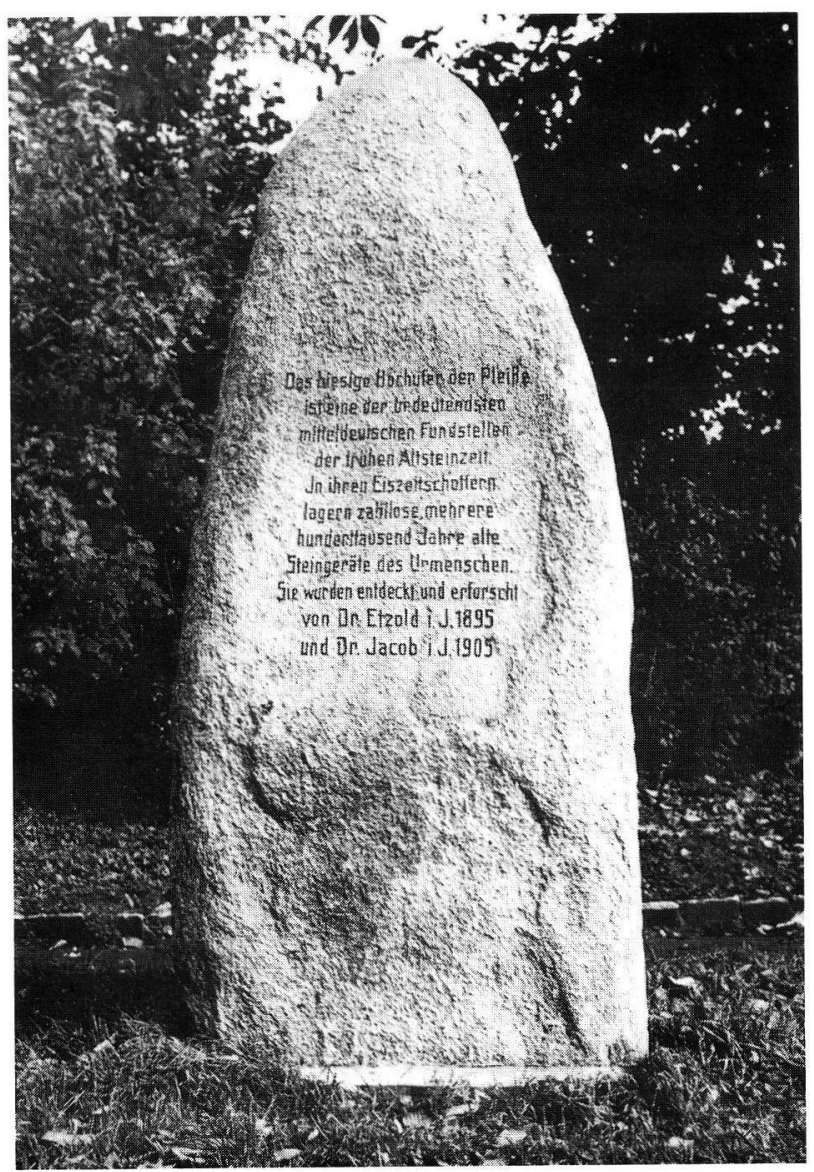

Abb. 16: Denkstein für die Altsteinzeit-Funde in Markkleeberg bei Leipzig und ihre Entdecker ETzold und ЈАсов. Foto freundlichst zur Verfügung gestellt.

$\mathrm{D}$ a n k. Nicht alle Kollegen, die mir wertvolle Hinweise gaben, kann ich hier nennen, doch bin ich H. Balmer (Zürich) und J.-P. Portmann (Neuchâtel) für ihre "Gedenktafel-Führungen“ zu besonderem Dank verpflichtet.

Für ergänzende Angaben wäre ich dankbar!

\section{Schriftenverzeichnis}

Arbeitskreis der K. F. Schimper-Realschule (Beiträge von H. GöTz, W. KосH, K. MäcdefraU, K. Wörs) (1967): Erinnerungsgabe zum 100. Todestag des Naturforschers K. F. Schimper. 44 S.; Schwetzingen.

Balmer, H. (1969): Jean de Cinarpentier. - Gesnerus, 26: 213-232; Aarau.

- (1970): Ignatz Venetz. - Gesnerus, 27: 138-168; Aarau.

- (1974): Louis Agassiz. - Gesnerus, 31: 1-18; Aarau.

- (1975): Louis Agassiz 1807-1873, der Mann und sein Werk. - Denkschr. Schweiz. Nat. Ges., 89: 9-20; Zürich.

- (1975): Edouard Desor und sein Landsitz Combe-Varin. - Gesnerus, 32: 61-86; Aarau.

- (1976): Geschichte der Naturwissenschaften in Aarau. - Gesnerus, 33: 108-120; Aarau. 
Böнм v. Böhmersheim, A. (1901): Geschichte der Moränenkunde. - Abh. Geogr. Ges. Wien, 3: 1-334; Wien.

Budmiger, G. (1980): Ein Stück Pioniergeschichte. F. J. Hugr und seine Begleiter im Rottal, 1830. - Die Alpen, 56: 1-2; Bern.

EissmanN, L. (1974): Die Begründung der Inlandeistheorie für Norddeutschland durch den Schweizer Adolph v. Monlot im Jahre 1844. - Abh. u. Ber. Naturkdl. Mus. „Mauritianum“, 8: 289-318; Altenburg.

HöLdER, H. (1960): Geologen-Denkmäler. - Nat. u. Volk, 90: 82-91; Frankfurt a. M.

- (1962): Geologen-Denkmäler. II. - Nat. u. Volk, 92: 322-330, Frankfurt a. M.

KaIser, K. (1975): Die Inlandeis-Theorie, seit 100 Jahren fester Bestand der Deutschen Quartärforschung. - Eiszeitalter u. Gegenwart, 26: 1-30; Öhringen.

Kuhn-Schnyder, E. (1975): Louis Agassiz als Paläontologe. - Denkschr. Schweiz. Nat. Ges., 89: $21-113$, Zürich.

Mariétan, I. (1959): La vie et l'oeuvre de l'ingénieur Ignace Venetz. - Bull. Murithienne, 76: $1-51$; Sion.

Portmann, J. P. (1962): Louis Agassiz, pionnier de la glaciologie. - Ann. Guébhard, 38: 239-248; Neuchâtel.

- (1967): A propos de Pierrabot. - Bull. Soc. Neuchât. Sci. Nat., 90: 291-292; Neuchâtel.

- (1975): Deux siècles de géologie à Neuchâtel. — Gesnerus, 32: 45-59; Zürich.

Renevier, E. (1877): Notice sur les blocs erratiques de Monthey. - Bull. Soc. Vaud. Sci. Nat., XV, 78: 105-116; Lausanne.

Schwarzbach, M. (1976a): Europäische Stätten geologischer Forschung. - 191 S.; Stuttgart (Wiss. Verlagsges.).

- (1976b): Der Eiszeit-Planet Erde. - Naturwiss. Rdsch., 29: 421-425; Stuttgart.

- (1979): Eiszeitgeologie für jedermann. - Naturwiss. Rdsch., 32: 65-66; Stuttgart.

- (1980a): Alfred Wegener und die Drift der Kontinente. - Gr. Naturforscher, 42, 160 S.; Stuttgart (Wiss. Verlagsges.).

- (1980b): Auf den Spuren von Alfred Wegener. - Der Aufschluß, 31: 504-509; Heidelberg.

WAGENBReth, O. (1978): Die Feuersteinlinie in der DDR, ihre Geschichte und Popularisierung. Schriftenr. geol. Wiss., 9: 339-369; Berlin.

Manuskript eingegangen am 6. 10. 1980. 
